\title{
El multiculturalismo en un juego de contextos estatales, regionales y globales en África*
}

\author{
Maguemati Wabgou**
}

Recepción: 8 de agosto de 2012 • Aprobación: 11 de diciembre de 2012

\section{Resumen}

En este artículo se hacen algunas referencias a la riqueza y vitalidad de las culturas de África que sirvan para cimentar políticas multiculturales en el continente. Esto lleva a abordar la problemática sobre la nación incluyente y el Estado en África, y a examinar los alcances de la búsqueda de la unidad nacional a partir de la diversidad cultural de los pueblos africanos; es decir, este artículo se enfoca en el análisis de las relaciones entre los Estados africanos y las diversidades culturales.

Palabras clave: cultura, diversidad cultural, África, nación inclusiva, unidad nacional.

Artículo de reflexión que el autor erige sobre el fruto de sus investigaciones pasadas en el grupo de investigación Migraciones y Desplazamientos.

* Profesor en el Departamento de Ciencias Políticas, Facultad de Derecho, Facultad de Ciencias Políticas y Facultad de Ciencias Sociales de la Universidad Nacional de Colombia, sede Bogotá. Responsable del grupo de investigación Migraciones y Desplazamientos (UNIJUS). Integrante del Grupo de Estudios Afrocolombianos (GEA-CES). Correo electrónico: mwabgou@unal.edu.co 


\section{Multiculturalism in a set of state, regional and global contexts in Africa}

\section{Abstract}

This paper makes some references to the richness and vitality of African cultures that serve to build multicultural policies in that continent. This leads to address the issue of inclusive nation and the state in Africa, and to examine the scope of the search for national unity from the cultural diversity of African peoples, that is, this paper focuses on the analysis of the relationships between African states and cultural diversities.

Keywords: culture, cultural diversity, Africa, inclusive nation, national unity. 


\section{Le multiculturalisme en un jeu de contextes étatiques, régionaux et mondiaux en Afrique}

\section{Résumé}

Dans cet article sont faites quelques références à la richesse et à la vitalité des cultures en Afrique qui soient utiles pour donner des bases à des politiques multiculturelles dans le continent. Ceci mène à aborder la problématique sur la nation inclusive et l'état en Afrique, et à examiner la portée de la recherche de l'unité nationale en partant de la diversité culturelle des peuples africains; c'est-à-dire, cet article est centré sur I'analyse des relations entre les États africains et les diversités culturelles.

Mots-clés: culture, diversité culturelle, Afrique, nation inclusive, unité nationale. 


\section{Introducción}

Un acercamiento al continente africano nos hace precisar que se trata de un espacio con muchas diversidades y contrastes culturales, sociopolíticos y económicos; situación que rompe con la visión reduccionista que tiende a referirse al continente como un conjunto de territorios con características globalmente homogéneas, lo que contribuye a reforzar las visiones sesgadas sobre este. Compuesta oficialmente de cincuenta y cuatro países, sin contar las demás identidades étnicas que reclaman su autodeterminación, África es un continente cuyas realidades suelen escapar al extranjero.

Con una superficie de $30.305 .053 \mathrm{~km}^{2}$, África cuenta con una población que ronda los 1000 millones de habitantes, en 2011 (precisamente, 1.037.524.058 de habitantes). Aparte de los idiomas oficiales (francés, inglés, portugués y español), herencia del pasado colonial, existen más de 1000 lenguas, lo que lo consolida como el continente más políglota del mundo. Estas lenguas pueden reagruparse en cuatro grandes grupos: 1) las lenguas afroasiáticas, habladas en África del este, África del norte, el Sahel y el suroeste de Asia; 2) las nilo-saharianas, habladas en el Chad, Etiopía, Kenia, Sudán, Uganda y el norte de Tanzania; 3) las de níger-congo, que pertenecen a la familia Bantú en el África central y parte del África del sur; y 4) las khoisanas, habladas en gran parte del África del sur.

El África del norte, globalmente poblada por los árabes, es marcada por el islam como religión dominante, aunque también se practica minoritariamente las religiones cristianas. El África subsahariana está mayoritariamente poblada por negroafricanos y marcada por prácticas religiosas denominadas religiones tradicionales africanas (RTA), lo que no excluye la presencia de minorías blancas y mestizas, igual que la adhesión a la religión musulmana.

En este contexto, el artículo busca mostrar la importancia de los valores ancestrales en el África contemporánea, marcada por las tensiones entre las tradiciones y la modernidad, pero con las persistentes diversidades étnicas que resisten al paso del tiempo en un mundo cada vez más globalizado. 
Asimismo, el texto intenta responder a la siguiente pregunta: ¿cuáles son las expresiones del multiculturalismo y sus transformaciones padecidas en los ámbitos socioculturales y sociopolíticos? Por tanto, el artículo hace unas precisiones conceptuales en torno al multiculturalismo: 1) antes de abordar el análisis del fenómeno del multiculturalismo, en África, en la época anterior a su colonización; 2) para luego presentar brevemente un mapeo sobre las dinámicas multiculturales en el África contemporánea.

\section{Precisiones conceptuales}

La realización de este análisis sobre la situación del multiculturalismo en África inicia con el debate en torno a la multiculturalidad, el multiculturalismo y la interculturalidad en las sociedades modernas en general, con el fin de hacer aclaraciones y precisiones conceptuales.

En efecto, la multiculturalidad se presenta como una condición de hecho, en la que confluyen diferentes entramados culturales con o sin reconocimiento jurídico o político. Debe ser diferenciada del multiculturalismo, entendido como el conjunto de políticas que se despliegan en el ámbito del derecho. Este multiculturalismo no es único ni estático, sino que debe entenderse al igual que la multiculturalidad - y en general la culturadesde una perspectiva metacultural, es decir, como una articulación histórica y contingente (no estática), asociada a un régimen de verdad establecido por relaciones de saber y poder:

La multiculturalidad debe entenderse como la emergencia de la diferencia y mismidad puntuada de cultural en regímenes de verdad y de experiencia en contextos sociales y situaciones concretos, objeto de disputas y disensos. La cultura y la multiculturalidad deben ser historizadas y eventualizadas; de ahí la relevancia de la categoría de (meta)cultura (Restrepo, 2004, p. 279). 
Por su parte, el multiculturalismo alude al reconocimiento de la diferencia (bajo los principios de igualdad y diferencia). En esa dirección, y de acuerdo con el Restrepo (2004), pensamos "el multiculturalismo como un hecho social global (p. 282). Es decir, el imaginario social, teórico y político contemporáneo se encuentra cada vez más interpelado por la constitución de sujetos y subjetividades políticas, así como por novedosos objetos de prácticas gubernamentales, a partir de las luchas por la diferencia cultural puntuadas por la etnicidad. De ahí que este multiculturalismo como hecho social global responda a un régimen de verdad particular, a una metacultura, que imagina la diferencia cultural de una manera muy particular: el otro étnico, constituido como efecto de la operación de dicho régimen de verdad.

Además, siguiendo a Hall, Restrepo (2004, pp. 277-281) presenta los diferentes contextos en que se puede generar su articulación y la forma en que esta se hace, sin desconocer los matices que se pueden presentar: 1) contexto conservador, en el que se fagocitan las diferencias culturales en un horizonte cultural dominante de la mayoría; se busca socavar las condiciones de existencia y de posibilidad de la multiculturalidad; 2) contexto liberal, en el que se da una diferenciación entre lo público y lo privado y se ve al individuo como un ciudadano racional, homogéneo y universal; así, lo cultural y sus manifestaciones se insertan en la esfera de lo privado; 3) contexto neoliberal, en el que el mercado se muestra como principio regulador de lo social y de lo político. La diferencia de los individuos se reconoce en el mercado, y desde este punto de vista, los problemas de la diferencia cultural están reflejados en el consumo privado, sin la necesidad de que exista una redistribución del poder y de los recursos; asimismo, el lugar del Estado es cada vez más marginal; 4) contexto formal comunitarista, en el que se asumen las diferencias formales entre grupos y se acuerdan derechos grupales para todos.

Una síntesis comparativa nos permite afirmar que el multiculturalismo es un hecho de orden jurídico y político, mientras que la multiculturalidad es un hecho social relacionado con las interacciones sociales enmarcadas en la cotidianeidad. Esta distinción es corroborada por Giménez (2003), que aporta ejemplos para delimitar los límites de ambos conceptos: 
Una cosa es que en un determinado país, o en una escuela, la diversidad cultural tenga una determinada presencia, intensidad y modos de expresión, y que las relaciones entre los sujetos en función de sus identidades y culturas sean unas $u$ otras (con conflicto manifiesto, latente o ambos; con mayor o menor relación de dominación y subordinación; con actitudes y comportamientos racistas o no, etc.), y otra cosa es que cada cual (autoridades, partidos políticos, líderes sociales, grupos de opinión, profesionales, etc.) considere que es lo mejor en cuanto a cómo abordar y tratar la diversidad sociocultural (pp. 2-3).

Sin embargo, no existe una única forma de multiculturalismo, sino varias, de las cuales se destacan el multiculturalismo conservador, el multiculturalismo liberal, el multiculturalismo neoliberal y el multiculturalismo formal comunitarista (Restrepo, 2004, pp. 279-280). De todos modos, el multiculturalismo es, en la actualidad, una realidad social global, con trasfondo político o jurídico, que se ha manifestado, en cierta medida, desde el colonialismo hasta la globalización neoliberal, pasando por la modernización y el desarrollismo.

En definitiva, el multiculturalismo opera mediante diversas políticas públicas de las diversidades y la diferencia, y se conecta de múltiples formas con articulaciones de alteridad (el "otro" como desplazado, inmigrante en situación de ilegalidad y legalidad, inmigrante o desplazado negro, chino, latino, hombre/mujer, empresario, pobre, etc.) que le han precedido y que continúan mutando según las lógicas de las transformaciones sociales o del cambio social:

Cuando se dice, por ejemplo, que una escuela es "multicultural", se puede estar diciendo que en ese centro escolar están escolarizados españoles y extranjeros, autóctonos e inmigrantes, gitanos y no gitanos (payos). Se está indicando con ello que es relevante la presencia de minorías étnicas, que hay una diversidad cultural notable, etc. Pero se puede estar diciendo -y así ocurre en países como Inglaterra, de amplia aplicación del multiculturalismo- que ese centro escolar responde a 
las normativas y orientaciones multiculturalistas, tratando de superar los currículums ocultos y etnocéntricos, organizando la escuela para que se vean reflejadas las distintas expresiones culturales, contratando a profesores bilingües y biculturales, etc. (Giménez, 2003, p. 4).

Es aquí donde surge la noción de interculturalidad, que promueve la convivencia en la diversidad, con base en los principios de igualdad, diferencia e interacción constructiva o positiva. La perspectiva de la interculturalidad busca desarrollar una interacción entre personas, conocimientos y prácticas culturalmente diferentes, para garantizar la transformación de las relaciones sociales y de las estructuras e instituciones públicas.

La interculturalidad se diferencia del multiculturalismo en la medida en que se presenta como un proyecto que cuestiona los diversos multiculturalismos, porque considera que estos se quedan en el reconocimiento de la diferencia en el marco de una jerarquía de poderes. Es más, se distancia del multiculturalismo por ser un proyecto que cuestiona sus diversas manifestaciones; pero, al criticarlos, centra el reconocimiento de la diferencia en el marco de una jerarquía de poderes:

La interculturalidad no se inscribe al reconocimiento de un Estado o una sociedad nacional de unos particularismos culturalistas [...] La interculturalidad no es solo el "estar" juntos, sino el aceptar la diversidad del "ser" en sus necesidades, opiniones, deseos, conocimiento, perspectiva, etc. (Mignolo, 2002a, p. 25, citado en Restrepo, 2004, p. 281).

En este sentido, la interculturalidad busca la pluriversalidad antes que la universalidad, teniendo en cuenta las múltiples relaciones de poder que posicionan globalmente unos particulares, naturalizándolos como "universales"; una pluriversalidad que asume seriamente las problemáticas de la diferencia y la historicidad de esta (Restrepo, 2004, p. 281).

De acuerdo con Giménez (2003), "la perspectiva intercultural está surgiendo básicamente tras la constatación de los límites, fracasos y errores 
en el campo del multiculturalismo, lo cual no debe ocultar los méritos y aportaciones de la perspectiva multiculturalista" (p. 4). De todos modos, el multiculturalismo y la interculturalidad se identifican como dos modalidades dentro del pluralismo cultural:

Tras unas primeras décadas en que la propuesta sociocultural pluralista de los años sesenta se concretó en elaboraciones y políticas multiculturalistas, desde finales de los ochenta han venido tomando fuerza los planteamientos interculturalistas en campos tan diversos como la educación, mediación, comunicación, trabajo social, filosofía, etc. [...] Por ello, y porque ambos planteamientos tienen en común los pilares del edificio propuesto desde el pluralismo cultural (valoración positiva de la diversidad, crítica de las propuestas de pérdida o resta cultural, igualdad y no discriminación por razones de diferenciación etnocultural de las personas, respeto al diferente, etc.), es por lo que consideramos más ajustado - y clarificador — ver el multiculturalismo y la interculturalidad como concreciones sucesivas del paradigma pluralista (Giménez, 2003, pp. 3-4).

Abogamos por la interculturalidad y en un alto grado el pluralismo cultural en las sociedades actuales, porque es la vía adecuada para emprender lineamientos y programas favorables a la solidaridad, integración y cohesión social que son necesarias para la construcción y consolidación de la nación.

Lo anterior lleva a afirmar que, en definitiva, la acción social es el motor de las interacciones sociales; esto es, las que implican tanto los nativos como los foráneos en un Estado determinado. De este modo, centramos nuestro análisis en el hecho social del multiculturalismo en África, buscando resaltar las formas como las interrelaciones de corte multicultural entre los africanos (individuos y colectividades) llevan a los individuos a participar en una dinámica interactiva en la que cabe la posibilidad de tensiones y conflictos políticos, con trasfondo étnico. 


\title{
Multiculturalismo en África precolonial:
}

\section{una perspectiva histórica}

La experiencia africana del multiculturalismo en la época precolonial es bastante limitada debido al hecho de que no existían Estados que lo promovieran. Sin embargo, en este continente, la multiculturalidad se ha expresado siempre a lo largo de la historia precolonial, teniendo en cuenta el hecho de que, tradicionalmente, la organización social secular presenta una estructuración en torno al grupo étnico. Esta estructura está regida por leyes y costumbres bajo el mando de un jefe que ocupa esta posición por reunir valores, habilidades, conocimientos empíricos, reconocidos por la mayoría de los integrantes del grupo social. Por ello, emerge como jefe del grupo étnico y se vuelve el garante de la cultura del pueblo o de la etnia. Asimismo, eludimos el uso del concepto grupos tribales identificados por su carácter cerrado y atrasado. Abogamos, más bien, por la referencia a los términos grupos étnicos, etnias o comunidades étnicas, caracterizados por su semejanza lingüística y su apertura a otros valores:

\begin{abstract}
Una etnia presenta características culturales (instituciones, idiomas, folclore, etc.) que la distinguen claramente de otros grupos. Tanto sus miembros como las poblaciones vecinas son conscientes de eso (Barbier, citado en François, 1993, p. 57).
\end{abstract}

Una definición parecida del concepto nos viene de Amin (1976): “La etnia —y no la raza, evidentemente- presupone una comunidad lingüística y cultural y una homogeneidad del territorio geográfico y sobre todo la consciencia de esta homogeneidad cultural" (p. 86).

En este contexto precolonial, África, marcada por prácticas ancestrales y diversidades culturales que se reflejan a través de modos y lógicas de pertenencia de sus habitantes a sus respectivos grupos étnicos, era un continente donde se practicaba la vida comunitaria con base en un fuerte sentido de solidaridades tradicionales y asociativas, derivadas del sentido de pertenencia a la familia extensa y a las asociaciones locales de cooperación, el apoyo recíproco y la ayuda mutua. Sin embargo, estas 
pautas de vida tradicional, comunitaria y ancestral fueron despreciadas por los exploradores, invasores, imperialistas y colonizadores europeos en esta zona "periférica", al calificar los pueblos autóctonos de "primitivos", "salvajes" (o imbuidos de "salvajismo"). Ello justificaba la necesidad europea de civilizar y educar a estas "tribus" africanas: cristianizar, colonizar y comercializar (teoría de las 3c).

A pesar de las resistencias de buena parte de los africanos al sistema político colonial, se produjo un aplastamiento de todas las formas de organización política (los reinos e imperios) existentes en aquella época. Asimismo, se ejerció una represión sistemática sobre organizaciones o sistemas políticos tradicionales, por ejemplo, el imperio de Songhaï (800 a 1591); el imperio de Malí (1200 a 1500); ei imperio de Dahomey (siglo XVII a 1906); el reino Oyo (siglo XIV a 1901); el imperio de Ghana (siglo XI al siglo XIII); el imperio Tuculeur (1820 a 1893); el impero Zulú (finales del siglo XVIII a 1879); el imperio Mossih (siglo XIV a 1896); el imperio Ashanti (siglo XVII a 1901). El único imperio que venció a los colonos en África fue el de Etiopía, bajo el mando del famoso emperador Menelik II, triunfador sobre los italianos en Adoua (1896)'. Al respecto, la observación de Boahen (1987b) nos parece de una relevancia sobresaliente:

En una fecha tan tardía como 1880, alrededor del $80 \%$ del continente africano estaba gobernado por los propios reyes, reinas, clanes y cabezas de linajes, en imperios, reinos, comunidades y organizaciones de diversos tamaños y formas [...] ¿Cuál fue la actitud de los africanos mismos con respecto al colonialismo, que implica, tal como ocurrió, un cambio tan fundamental en la naturaleza de las relaciones que existían entre ellos y los europeos desde hacía trescientos años? [...] La respuesta es completamente clara e inequívoca: una abrumadora mayoría de las autoridades y líderes africanos se opuso vehementemente a este cambio y expresó su determinación de mantener el statu

1 Véase para más detalles sobre las resistencias africanas, cruelmente reprimidas mediante conquistas militares, francesas e inglesas (1885-1902), que solían culminar con la deportación de los reyes o líderes africanos a tierras extranjeras (Uzoigwe, 1987, pp. 58-67). No descartamos las políticas racializantes practicadas por el sistema colonial portugués. 
quo, y, por encima de todo, de conservar su soberanía e independencia (pp. 23-25).

Pero, afortunadamente, buena parte de todas estas formas ancestrales y tradicionales de vida han sobrevivido a la agresión colonial de los europeos y persisten en el África contemporánea, estirada entre las tradiciones y la modernidad.

\section{Situación del multiculturalismo en África hoy}

Además, la existencia de las familias extensas y los clanes en el África tradicional y precolonial favorece el contacto y la interacción entre miembros de estos grupos sociales, destacando el rol primordial del parentesco en la organización social y política. Cualquier expresión social de índole comunitaria se desarrollaba desde las familias extensas y clanes, puesto que sus integrantes son descendientes de un ancestro común, lo que contribuye al mantenimiento de una cierta armonía social.

En términos poblacionales e identitarios, se observa que, generalmente, el norte de África está poblada por los árabes, y la parte subsahariana está dominada poblacionalmente por los negros; Sudáfrica, por su parte, cuenta con una significativa población blanca y mestiza. Esta anotación se refiere a la imposibilidad de hacer una delimitación drástica de índole poblacional, cultural y religiosa entre estas zonas territoriales, ya que África subsahariana, en general, se define como todos los países al sur del Sahel. Sin embargo, destaca por la imprecisión en sus delimitaciones geográficas, puesto que es una región del continente que encierra grandes contrastes. Señala Founou-Tchuigoua (1995):

[Sus límites] no son muy precisos. Se trata de una denominación que sustituyó a la del África negra, diferenciada del África blanca del mediterráneo. La toma de conciencia del carácter multirracial, no solo de Sudáfrica y de Namibia, sino también de ciertos países de Sahel, en particular, hizo que el nuevo concepto se 
adoptará con facilidad. Contra lo que puede sugerir la opinión corriente, subsahariano no se opone a árabe, ya que Mauritania, Sudán y Somalia son consideradas por lo general como parte integrante del grupo subsahariano. Sudáfrica no suele incluirse en este grupo, por su apartheid y su nivel de industrialización, el más alto del continente. Así, el fin del apartheid no hará de Sudáfrica un país subsahariano cualquiera. El desmantelamiento efectivo del apartheid y sus efectos sobre África austral justificarán por mucho tiempo todavía un tratamiento especial para este país. Es evidente que el espacio delimitado no tiene unidad económica, y jamás formó una unidad política, antes, durante o después de la colonización. Ciertamente, la negritud y el panafricanismo imprimieron en las mentalidades el concepto de la unidad cultural precolonial y las aspiraciones a la unidad política, a la formación de grandes subregiones fundadas en la geografía y en esta unidad cultural precolonial, reforzadas por experiencias coloniales comunes o similares (pp. 141-142).

Partiendo de uno de los núcleos centrales a la sociedad africana: el grupo étnico, consideramos que las formas de expresión del multiculturalismo en el África contemporánea se relacionan con las prácticas religiosas, las expresiones lingüísticas y artísticas, folclóricas y la cosmovisión.

\section{Prácticas religiosas}

Desde la perspectiva de las religiones tradicionales (RTA), en el África subsahariana cobran mayor importancia las ceremonias o rituales que se vuelven garantes del bienestar espiritual de su clan, aunque la naturaleza de los sacrificios y ofrendas (gallina, cabra, cosechas) esté determinada por el adivino, quien se dedica a consultar los oráculos. Estas creencias y prácticas religiosas en torno al ancestro común (cultos ancestrales) y al líder del clan avivan las energías de los pueblos y favorecen la cohesión de la comunidad-clan; son expresiones y vehículos de la espiritualidad de los pueblos; traducen las realidades sociales vividas; estructuran identidades en torno a la tierra o al aire geográfico en el que se reside, esto es, a la 
espiritualidad se une la territorialidad. Las ceremonias o cultos congregan a todos los miembros del clan, incluso a quienes están en otros territorios.

Las religiones tradicionales africanas constituyen cultos religiosos practicados por la mayoría de hombres y mujeres del África subsahariana. En África del oeste, por ejemplo, se presentan bajo varias denominaciones: vodú, tîngban, etc. Profesan la creencia en un dios supremo (creador) cuya supremacía y lejanía es tan notoria que uno no puede dirigirse directamente a él. Quien quiere solicitar algo a dios, tiene que pasar por uno o varios intermediarios, representados por los ancestros o creadores de la sociedad, de la misma forma que el ser o dios supremo es creador del cosmos. Creen que existen otras pequeñas deidades cuyo espíritu expresa fenómenos naturales, tales como el viento, el río, el mar, el árbol o ciertos animales. Estos intermediarios tienen un papel indispensable en las religiones tradicionales, ya que mediante una ofrenda se espera un mejor acercamiento a los ancestros o a los dioses, quienes podrían suavizar sus posturas e intercederían ante el dios supremo a favor de quienes hacen las ofrendas. Igualmente, se realizan ofrendas a los dioses cuando una persona, un clan o una familia sufre de una calamidad inexplicable: la idea es que el pequeño dios pueda conjurar los espíritus maléficos que hayan causado el daño que les afecta.

Las ceremonias no se hacen solamente en momentos de desgracias, sino también en momento de felicidad: por ejemplo, cuando los agricultores obtienen buenas cosechas o después del nacimiento de un niño. Estos ritos, cultos y ceremoniales pueden cumplirse en cualquier momento, constituyéndose en el medio más materializado de comunicación entre el Dios supremo y los mortales (mujeres, hombres y niños), a través de sus deidades. Cabe precisar que en países del África subsahariana se encuentran minorías de adeptos de la religión musulmana, aunque esto no excluye que haya países con población mayoritariamente musulmana como el caso de Senegal.

En el África del norte, la religión musulmana ocupa el primer lugar como culto religioso, lo que no excluye la práctica de la religión cristiana por una minoría. Además, es necesario diferenciar el islam —religión practicada 
en escenarios privados por personas que creen en las palabras del profeta Mahoma y están plasmadas en el Corán-y el islamismo — que reúne a los sectores más radicales de la religión, identificados hoy en día como "salafistas"—.

De todos modos, lo que apuntamos en el marco del análisis del multiculturalismo en África es la coexistencia de las distintas prácticas religiosas mencionadas en múltiples escenarios sociopolíticos; más aún cuando observamos que entre los grupos musulmanes existen muchas prácticas, similares a las de las RTA, tales como los conjuros o encantamientos y la confección de talismanes y otros artificios mágicos para dar suerte u otorgar protección. El imam o jefe religioso será consultado por los adeptos que tienen problemas. Por otra parte, el cristianismo, al estar asociado con la perfeccionada técnica europea, tiene una reputación parecida para el pueblo africano y cohabita con instituciones sincretistas y mesiánicas que desempeñan casi la misma función religiosa. Por consiguiente, es frecuente encontrar a africanos adeptos a la fe tradicional (RTA), que compaginan su fe y ritos con los cristianos. Una de las razones de esta actitud religiosa es que los actores creen que con estas formas más eficaces de protección supernatural del cristianismo pueden incrementar sus medios de protección.

Estas son características afines al multiculturalismo, en la medida que desbordan los límites de la multiculturalidad que se refiere a una condición de hecho en el marco de la cotidianidad y captan un mayor interés político y jurídico.

\section{Expresiones lingüísticas}

Como lo enunciamos en la introducción del presente trabajo, África es considerado el continente más políglota del mudo, teniendo en cuenta que allí se hablan más de 1000 lenguas, aparte de los idiomas oficiales (francés, inglés, español y portugués). De estas lenguas se suele apostar por una como lengua nacional que se enseña en las escuelas y cobra un carácter oficial, ya que se habla en varios sectores de la población. 
Lo interesante de estas lenguas nacionales es que, teniendo en cuenta que el analfabetismo afecta todavía a varios sectores de las poblaciones, esta lengua se suele hablar por personas analfabetas, lo que le legitima el uso como lingua franca, paralelamente a la lengua oficial que suele ser uno de los mencionados idiomas europeos de las exmetrópoli colonizadora.

En este sentido, la idea suele consistir en impulsar la unidad nacional a partir de esta lengua nacional. Sin embargo, existen casos atípicos como el de Togo, del dictador Gnassingbé Eyadema, donde se impulsaron dos lenguas nacionales en vez de una. Asimismo, no cabe duda de que estas medidas hacen parte del conjunto de políticas lingüísticas que promueven el multiculturalismo, al impulsar interacciones sociales entre miembros de distintos grupos étnicos, más allá del idioma oficial cuyo aprendizaje se limita solo en el marco de la educación formal. No sobra mencionar la importancia del swahili ${ }^{2}$ en el contexto actual del continente africano, en la medida que es promovido por la Unión Africana como una lengua panafricanista:

También conocido como kisuahili, el suahili comenzó a expandirse mediante extensas interacciones entre africanos de la región oriental con los del océano Índico tal como las islas Comores y Madagascar; teniendo en cuenta que el comercio y las migraciones ayudaron a difundir la lengua entre naciones del sureste africano tales como Malawi y Tanzania. Es discutible cuánta gente habla suahili como primera o segunda lengua,

2 Además, Varela (2006) aporta las aclaraciones siguientes: "El swahili se distingue, entre otros aspectos, por ser la lengua de uso más extendido en la parte este de África. Más de 40 millones de personas la hablan, ya sea como lengua materna o como segunda o tercera lengua. Además de ser la lengua oficial de Kenia y Tanzania, es una lengua importante en Malawi, Mozambique, República Democrática del Congo, Somalia y Uganda. A través de los movimientos migratorios de mano de obra, el swahili ha llegado también hasta Zimbabwe y Sudáfrica. Existen importantes comunidades swahili hablantes en países como el Reino Unido, Holanda, Alemania, Canadá y Estados Unidos. En este último país, el swahili ha sido adoptado por las comunidades afroamericanas como la lengua panafricana por excelencia. Desde que fue diseñado el currículo, para la selección de esta lengua se tomó en cuenta el hecho de que en la hoy desaparecida Organización de la Unidad Africana, el swahili era una de las lenguas oficiales. Hoy en día es una de las lenguas de trabajo en la Unión Africana, además de ser la lengua africana más difundida en ámbitos universitarios, por ejemplo en numerosas universidades de Europa y Asia. En universidades de Estados Unidos es la lengua que suma la mayor cantidad de cursos (cátedras). El estudio del swahili se facilita debido a la gran cantidad de materiales didácticos y diccionarios para personas que hablan inglés, francés, italiano, alemán y español, entre otros idiomas. Existe una gran producción literaria en lengua swahili, entre los autores más relevantes pueden ser citados Shabaan bin Robert, Euphrase Kezilahabi y Mohamed Sulaiman" (p. 10). 
puesto que las más conservadoras estimaciones hablan de 45 millones, mientras que otras señalan unos 60 millones de personas en África del este y central, particularmente en Tanzania, Kenia, Uganda, Somalia, Mozambique, Malawi, Rwanda, Burundi, Zambia y la República Democrática del Congo. En la actualidad, el suahili es una lengua oficial de la Unión Africana (UA), porque se considera que es parte de la identidad africana y se espera que en el futuro se convierta en "una lengua pan-africana". Las otras lenguas oficiales de la Unión Africana son el inglés, el francés, el árabe, el portugués y el español (Wabgou, 2008, p. 2).

\section{Expresiones folclóricas}

En el marco de las diversidades culturales de los pueblos africanos, se encuentran varias prácticas folclóricas en términos de prácticas ancestrales, cantos, ritmos, músicas, bailes, danzas y formas vestimentarias tradicionales, que coexisten de forma pacífica y enriquecen el panorama folclórico de las comunidades tanto en las zonas rurales como en las urbanas. Hablar de las músicas africanas implica señalar que las expresiones musicales en el África son muy sensuales, espirituales y enérgicas: son expresiones de alegría para celebrar éxitos, o de tristeza para aliviar penas comunales o personales. En general, existen aires musicales contemporáneos en boga que traspasan los límites de las fronteras de los países africanos para estar compartidos por la nueva generación en distintos países. Al respecto, señalamos que aparte del reggae jamaicano, adoptado por artistas africanos (los marfileños Alpha Blondy, Tiken Jah Fakoly, entre otros), con carácter reivindicativo también se encuentra el rap africano (de los grupos Djanta Kan de Togo, Magic Black Men de Malí, Faso Kombat de Burkina-Faso, Rap'Adio y Positive Black Soul de Senegal; los raperos Pee Frois, Abass Abass, Didier Awadi y PBS-Radical de Senegal; el rapero Dalai Ka de Benin, y el grupo Kilio Cha Haki de Kenia). Además, se destacan el soukouss del Congo Democrático y el resto de África central y occidental, que se disfruta a lo largo y ancho del continente africano, liderado por artistas como Rochereau, Franco, Mbilia Bel, Loketo, Papa Bemba, Koffi Olomide, Kanda Bongo Man, Wenge Musica; el mbalax de 
Senegal, encabezado por Yousou N’Dour; la música madingue o griot de los países sahelianos del África occidental, liderada por Salif Feita y Baba Maal; y el raï de Algeria y el resto del norte de África, liderado por Khaled Hafla, entre otros. Estos y otros aires musicales y estilos de danza afectan las vidas y experiencias de hombres y mujeres africanos en contextos nacionales, regionales e internacionales, marcados por el multiculturalismo.

Los instrumentos musicales más tradicionales utilizados producen sonidos diferentes. Se destacan el tambor, también conocido como djembé, y la kora. Antiguamente, el tambor era el medio privilegiado de comunicación entre dirigentes y pueblos: cuando el jefe del reino o de la aldea quería anunciar una noticia al pueblo, recurría a los tambores. Los antepasados africanos utilizaban el tambor para comunicarse y danzar en los días de fiestas; los tambores suenan a la hora de venir al mundo y a la hora de despedirse de la vida: convocan a la unidad. Las músicas africanas constituyen un medio para la transmisión de conocimientos y valores; suelen combinarse con habla, danzas y artes visuales para crear representaciones similares al mensaje contenido en las canciones. Lo que nos interesa es mostrar que todas estas formas musicales acompañadas de bailes o danzas específicas enseñan la cotidianidad.

\section{Artes africanas}

Señalamos las actividades creativas que tienen como referencia manifestaciones socioculturales subsaharianas como las máscaras ${ }^{3}$, las estatuas, las piedras pintadas, las figuras pintorescas, animales o forestales, los collares, las telas con sus coloridos diseños. Todo ello constituye el modo en el que la gente subsahariana se halla inserta. Mediante estas representaciones reflejan escenas cotidianas de caza, del mercado, en plazas públicas; expresan diversas formas de ver el mundo circundante. Son conocidas las artes antiguas en Malí (los de Tombuctú, los Dogón,

3 Cabe mencionar la omnipresencia de las máscaras en las ceremonias públicas y de carácter religioso en las sociedades africanas. Estas características las convierten en un objeto mágico mediante el cual las representaciones de espíritus en formas de animales $u$ otros elementos de la naturaleza sirven para invocar a las divinidades (incautaciones), con el fin de rendirles homenajes, o simplemente recordar al pueblo su discreta presencia en la comunidad, al velar en ella. 
etc.), Nigeria (los hausa, ifés, igbos, etc.), Ghana (ashanti, kan, ewé, etc.); las pinturas rupestres en el desierto del Sahara y del África austral (desierto kalahari). Y en el resto de África, el delta del Nilo en la región de Nubia y el Valle del Nilo (Egipto); las pinturas rupestres de los montes Tassili N'Ajjer (Argelia), en el desierto del Sahara y del África austral. Las obras artísticas contemporáneas siguen el rumbo de la multiculturalidad y el multiculturalismo, incluyendo las obras de reivindicaciones, protestas y resistencias contra las dictaduras cuyo objetivo final es la defensa de los derechos de los ciudadanos, más allá de su pertenencia a grupos étnicos y distintas orientaciones políticas.

\section{Cosmovisión africana}

Se refiere a las formas de pensar, vivir y percibir su entorno material e inmaterial (filosofías). En general, permite dinamizar el relacionamiento y entrelazamiento entre distintas comunidades, ya que, por ejemplo, permite que no se rompan los lazos de la comunidad de suelo y la comunidad de fe que trasciende la territorialidad y une a unos y otros. En el África subsahariana, principalmente, la cosmovisión suele impactar a los miembros de las familias, a sabiendas de que la noción de familia va junto con la del clan. Este último es un referente esencialmente organizacional desde el punto de vista social, político, territorial y religioso. Es una entidad que reagrupa a todas las personas que creen tener un mismo ancestro mítico en común. Para que se constituya el clan, cada reagrupamiento familiar tiene que fundamentarse en tres pilares o realidades sociales que sientan sus bases: la comunidad de sangre (descendencia de un ancestro común), la comunidad de fe (culto a un ancestro común) y la comunidad del suelo (domicilios o casas en el mismo territorio ancestral). En otros términos, constituyen un conjunto de familias extensas que comparten un mismo territorio; lo que determina y condiciona su forma de ver y concebir el mundo. 


\section{A modo de conclusión: reflexiones finales sobre los retos y desafíos del multiculturalismo en África}

Aludimos a los cambios producidos por las Constituciones de los Estados modernos africanos derivados del colonialismo y las valiosas luchas para la independencia en la diversidad cultural africana y su manifestación en los contextos políticos, culturales y sociales. Con el surgimiento de los Estados modernos africanos, mayoritariamente en los años sesenta, se observa que sus respectivas Constituciones impulsan un conjunto mayor de reformas que propenden a la salvaguardia de la diversidad étnica y cultural en el marcado de un Estado unitario y centralista, aun si en los discursos políticos se aboga reiterativamente por la construcción de la nación que no necesariamente nació con los Estados: se trata de una nación multicultural y pluriétnica. Sin embargo, este aliento y afán ha permitido algunos excesos y excentricidades muy marcadas por el occidentalismo y la influencia de este sobre el pensamiento africano y la forma de organización política en África. Al respecto, Babalola Yai (2004) muestra cómo en África las Constituciones actuaban más bien en contra de las diversidades histórico-culturales, por efecto de la repartición regional que las potencias europeas hicieron en el continente durante el último decenio del siglo XIX, hasta el decenio de 1960. Sin embargo, el autor también insiste en que la ancestralidad había permitido contrarrestar o detener parte de los efectos perversos del colonialismo. Por ejemplo, la religión yoruba multiplicó las diversidades identitarias, al ofrecerle a cada persona la tutela y el culto particular de los orishas de sus aldeas respectivas, sus familias extensas, sus clanes y sus personas - como individuos y partes de la colectividad-, al identificar la atomización derivada de la colonización y las deidades mediante prácticas complejas de adivinación. Esta situación nos lleva a plantear una serie de problemáticas que se constituyen en limitaciones del florecimiento del multiculturalismo y de la interculturalidad y el pluralismo cultural en África.

Por un lado, la cuestión del reordenamiento territorial de la nación persiste como una seria limitación a la consolidación del multiculturalismo. La construcción de la nación, muy débil, en todos estos jóvenes Estados del África se vuelve una prioridad en medio de gobiernos "democráticos", 
autócratas, populistas y dictatoriales. Por la misma lógica del surgimiento del Estado-nación en África poscolonial, se observa la intolerancia étnica de parte de los gobiernos centrales que no dan relevancia al carácter multiétnico de la nación y se quedan con el sentido heredado de la Revolución francesa: sentido de pertenencia a un mismo Estado, una misma identidad territorial con los mismos objetivos. Menospreciando el carácter voluntario de la nación —unión voluntaria y soberana de individuos autónomos e iguales-, los líderes africanos insisten desesperadamente en el desarrollo de conciencia política nacional. Mientras tanto, el poder central suele carecer de iniciativas, políticas e instituciones apropiadas para la educación de los pueblos en temas afines al civismo, ciudadanía, cultura política, participación, y se niega a reconocer los derechos políticos de los grupos étnicos que están al margen del ejercicio del poder o de la vida política creada o impuesta por el régimen dictatorial.

Por otro lado, surgen los conflictos armados y el clientelismo como partes del modus operandi de escenarios políticos africanos. Las formas de relación de los actores armados con las distintas expresiones de las diversidades culturales y regionales tiende a instrumentalizarlas para fines políticos: surgen interrogantes sobre cómo las conciben; si las aprecian y respetan; si las instrumentalizan para sus reivindicaciones con fines meramente políticos; si la miran con maniobras maniqueas (prevención y sospecha). ¿Qué consideración tienen los actores bélicos — tanto los señores de la guerra como el ejército- sobre las consecuencias de estos conflictos en términos de producción de grandes flujos o desplazamientos forzados que incurren los caminos del miedo, derivado del terror de la guerra, siendo los miembros de las comunidades sacados de sus territorios ancestrales?

En estos contextos multiculturales, bajo la amenaza del estallido de conflictos políticos y en medio de la crisis socioeconómica, la garantía y el respeto de los derechos humanos de las personas se vuelve un elemento fundamental para la promoción del multiculralismo en África, sin desconocer las particularidades de Sudáfrica, por ejemplo (McKinne y Crain, 2010), igual que la posibilidad de tensiones entre los requisitos universalistas de derecho internacional humanitario, la ciudadanía multicultural y las tradiciones o las visiones y prácticas tradicionalmente locales. 
Sin duda, es fundamental seguir apostando por la apertura hacia el multiculturalismo y el inicio del sendero hacia el interculturalismo, pasando del plurietnicismo (o la multietnicidad) al multiculturalismo efectivo, la interculturalismo y el pluralismo cultural, con el apoyo y acompañamiento decidido del aparato estatal en un mundo cada vez más global. La puesta en marcha de la interculturalidad es uno de los mayores retos a los cuales se enfrentan los Estados africanos, al igual que la mayoría de las sociedades modernas, porque se plantea la posibilidad de superar los límites de las instituciones estatales, con el fin de incluir a una mayor parte de las personas y la sociedad civil (grupos étnicos, organizaciones no gubernamentales y locales, sindicatos, academia, etc.) en los procesos de elaboración de las políticas favorables a una mayor interacción social y tolerancia; lo que podría ser de gran utilidad para reforzar los lazos y conexiones entre de África y Afroamérica.

\section{Referencias}

Amin, S. (1976). Sobre el desarrollo desigual de las formaciones sociales. Barcelona: Anagrama.

Babalola Yai, O. (2004). Religión y nación multicultural: un paradigma del África precolonial. En J. Arocha (comp.) Utopía para los excluidos: multiculturalismo en África y América Latina (pp. 19-87). Bogotá: Universidad Nacional de Colombia, Facultad de Ciencias Humanas, Centro de Estudios Sociales.

Boahen, A. A. (1987). África y el desafío colonial. En Historia general de África (vol. VII: África bajo el dominio colonial, 1880-1935; pp. 23-40). Madrid: Tecnos.

François, Y. (1993). Le Togo. París: Karthala.

Founou-Tchuigoua, B. (1995). El África subsahariana. La cuartomundización en crisis. En S. Amin y P. G. Casanova (dirs.) La nueva organización capitalista mundial vista desde el Sur. Mundialización y acumulación (vol. l; pp. 141-208). Barcelona: Anthropos. 
Giménez, C. (2003). Pluralismo, multiculturalismo e interculturalidad: propuesta de clarificación y apuntes educativos. Recuperado de www.cesdonbosco. com/revista/impresa/8/estudios/texto_c_gimenez.doc

McKinney, C. y Soudien, C. (2010). IALEI Country Report Multicultural Education in South Africa. University of Cape Town. Recuperado de http://www.intlaIliance.org/fileadmin/user_upload/documents/Conference_2010/NP-SA.pdf

Restrepo, E. (2004). Biopolítica y alteridad: dilemas de la etnización de las colombias negras. En E. Restrepo y A. Rojas (Eds.) Conflicto e (in)visibilidad. Retos en los estudios de la gente negra en Colombia (pp. 279-320). Popayán: Universidad del Cauca.

Uzoigwe, G. N. (1987). La división y conquista europeas de África: visión general. En Historia general de África (vol. VII: África bajo el dominio colonial, 18801935; pp. 41-67). Madrid: Tecnos.

Varela, H. (2006). Los estudios de África en El Colegio de México. Estudio histórico. Ponencia presentada en el Seminario Internacional sobre los Estudios Africanos en América Latina. Herencia, Presencia y Visiones del Otro (mimeo). Salvador de Bahía.

Wabgou, M. (2008). Kenia: ante los desafíos del multipartidismo y la democracia liberal. Nova Africa, 23, 25-38. Barcelona: Centro d'Estudis Africans. 